\title{
Improving Electron Mobility of Tetraphenylethene- Based AIEgens to Fabricate Nondoped Organic Light-Emitting Diodes with Remarkably High Luminance and Efficiency
}

Gengwei Lin, ${ }^{\dagger}$ Huiren Peng, ${ }^{*}$ Long Chen, ${ }^{+}$Han Nie, ${ }^{\dagger}$ Wenwen Luo, ${ }^{\dagger}$ Yinghao Li, ${ }^{\dagger}$ Shuming Chen, * Rongrong Hu, ${ }^{\dagger}$ Anjun Qin, ${ }^{\dagger}$ Zujin Zhao, ${ }^{* \dagger}$ Ben Zhong Tang ${ }^{*} \$ \zeta$

${ }^{\dagger}$ State Key Laboratory of Luminescent Materials and Devices, South China University of Technology, Guangzhou 510640, China

${ }^{\ddagger}$ Department of Electrical and Electronic Engineering, South University of Science and Technology of China, Shenzhen, Guangdong 518055, China

$\S$ Department of Chemistry, The Hong Kong University of Science \& Technology, Clear Water Bay, Kowloon, Hong Kong, China

$\zeta^{\zeta}$ Hong Kong Branch of Chinese National Engineering Research Center for Tissue Restoration and Reconstruction, Hong Kong, China

*Corresponding Author: E-mail: mszjzhao@scut.edu.cn, tangbenz@ust.hk, chen.sm@sustc.edu.cn. 


\section{Experimental details}

Materials and instruments. Tetrahydrofuran (THF) was distilled from sodium benzophenone ketyl under dry nitrogen immediately prior to use. Compounds $2^{1}, 4^{2}, 7^{3}$ and $8^{3}$ were prepared according to the literature procedures. All the other reagents and solvents were purchased from commercial sources and used as received without further purification. NMR spectra were obtained on a Bruker AV 500 or 600 spectrometer in deuterated chloroform or dichloromethane using tetramethylsilane (TMS; $\delta=0$ ) as internal reference. High resolution mass spectra (HRMS) were recorded on a GCT premier CAB048 mass spectrometer operating in MALDI-TOF mode. The ground-state geometries were optimized using the density functional with the B3LYP hybrid functional at the basis set level of 6-31G(d). All the calculations were performed using the Gaussian 09 package. UV-visible absorption spectra were measured on a SHIMADZU UV-2600 UV-vis spectrophotometer. Fluorescence spectra were recorded on a Horiba FluoroMax-4 spectrofluorometer. Fluorescence quantum yields were measured using a Hamamatsu absolute photoluminescence quantum yield spectrometer C11347 Quantaurus-QY. Thermogravimetric analysis (TGA) analysis was carried out on a TA TGA Q5000 and differential scanning calorimetry (DSC) analysis was performed on a DSC Q1000 under dry nitrogen at a heating rate of $10{ }^{\circ} \mathrm{C} \mathrm{min}^{-1}$. Cyclic voltammograms were measured on a CHI610E A14297. The CV measurement was conducted in dichloromethane with $0.1 \mathrm{M}$ tetrabutylammonium hexafluorophosphate as the supporting electrolyte at a scan rate of $50 \mathrm{mV} \mathrm{s}^{-1}$, using platinum as the working electrode, saturated calomel electrode (SCE) as the reference electrode, and platinum wire counter electrode. The 
SCE reference electrode was calibrated using the ferrocene/ferrocenium $\left(\mathrm{Fc}_{\mathrm{Fc}}{ }^{+}\right)$redox couple as an external standard.

Devices fabrication. Multilayer OLEDs were fabricated by the vacuum-deposition method. Organic layers were deposited by high-vacuum $\left(5 \times 10^{-4} \mathrm{~Pa}\right)$ thermal evaporation onto a glass $(3 \mathrm{~cm} \times 4 \mathrm{~cm})$ substrate pre-coated with an indium tin oxide (ITO) layer. 1,4,5,8,9,11Hexaazatriphenylenehexacarbonitrile (HATCN) was used as the hole-injecting layer, $N, N$ '-bis-(1naphthalenyl)- $N, N^{\prime}$-bis-phenyl-(1,1'-biphenyl)-4,4'-diamine (NPB) was utilized as the hole-transporting layer, TPE-TPAPBI and TPE-DPBI were employed as the light-emitting layers, 1,3,5-tris(1-phenyl-1Hbenzimidazol-2-yl)benzene (TPBi) was applied as the electron-transporting layer and LiF/Al was chosen as the cathode. All organic layers were deposited sequentially. Thermal deposition rates for the organic materials, $\mathrm{LiF}$ and $\mathrm{Al}$ were $0.5,0.5$ and $1 \AA \mathrm{s}^{-1}$, respectively. The active area of each device was $12 \mathrm{~mm}^{2}$. The electroluminescence spectra were measured on a Hitachi MPF-4 spectrofluorometer. The current density-voltage $(J-V)$ characteristics of the OLEDs were recorded on a Keithley 2400 Source Meter. The current density-voltage-luminance curves $(J-V-L)$ characterizations were carried out with a 3645 DC power supply combined with a 1980A spot photometer and they were recorded simultaneously. All measurements were done at room temperature under ambient conditions.

Fluorescence Lifetime. The time-resolved fluorescence spectra were recorded on a Hamamatsu C11367-11 Quantaurus-Tau time-resolved spectrometer. Decay of the PL intensity $(I)$ with time $(t)$ was 
fitted by a double-exponential function (Equation 1), and the mean lifetime was calculated according to Equation 2.

$$
\begin{gathered}
I=I_{0}+A_{1} e^{-t / \tau_{1}}+A_{2} e^{-t / \tau_{2}} \\
\langle\tau\rangle=\frac{A_{1} \tau_{1}+A_{2} \tau_{2}}{A_{1}+A_{2}}
\end{gathered}
$$

Synthesis. 4'-(2-(4-Bromophenyl)-1,2-diphenylvinyl)-N,N-diphenyl-[1,1'-biphenyl]-4-amine (9). Into a $250 \mathrm{~mL}$ two-necked round bottom flask with a reflux condenser were placed 2 (2.94 g, $6.0 \mathrm{mmol}), 4$ (867 mg, $3.0 \mathrm{mmol}), \mathrm{Pd}\left(\mathrm{PPh}_{3}\right)_{4}(104 \mathrm{mg}, 0.09 \mathrm{mmol})$ and $\mathrm{Na}_{2} \mathrm{CO}_{3}(954 \mathrm{mg}, 9.0 \mathrm{mmol})$. The flask was vacuumed and purged with dry nitrogen by three times. THF $(80 \mathrm{~mL})$ and deionized water $(20 \mathrm{~mL})$ were then injected into the flask. The reaction mixture was refluxed for $12 \mathrm{~h}$ under nitrogen atmosphere. After cooling to room temperature, the mixture was poured into water and extracted with dichloromethane. The combined organic extracts were dried over anhydrous magnesium sulfate, and subsequently filtered and evaporated the solvent. The crude product was purified by silica-gel column chromatography using petroleum ether/dichloromethane as eluent. Compound 9 was obtained as green solid in $70 \%$ yield (1.37 g). ${ }^{1} \mathrm{H}$ NMR (500 MHz, $\mathrm{CDCl}_{3}$ ), $\delta$ (ppm): 7.48-7.43 (m, 2H), 7.38-7.32 (m, 2H), 7.28-7.22 (m, 6H), 7.16-7.01 (m, 20H), 6.96-6.90 (m, 2H). ${ }^{13} \mathrm{C} \mathrm{NMR}\left(125 \mathrm{MHz}, \mathrm{CDCl}_{3}\right), \delta(\mathrm{ppm}): 147.6,147.2,147.1$, $143.5,143.4,143.3,142.8,142.0,141.9,141.3,139.6,138.6,138.4,134.5,134.4,133.0,131.7,131.3$, $130.9,130.8,129.3,127.9,127.8,127.7,127.5,126.7,126.6,125.8,125.7,124.4,124.0,122.9,120.5$, 120.4. HRMS $\left(\mathrm{C}_{44} \mathrm{H}_{32} \mathrm{BrN}\right): m / z 653.1713\left[\mathrm{M}^{+}\right.$, calcd 653.1718]. 
4'-(1,2-Diphenyl-2-(4'-(1-phenyl-1H-benzo[d]imidazol-2-yl)-[1,1'-biphenyl]-4-yl)vinyl)-N,N-

diphenyl-[1,1'-biphenyl]-4-amine (TPE-TPAPBI). This compound was synthesized by a similar method to that for compound 9, in which $9(1.31 \mathrm{~g}, 2.0 \mathrm{mmol}), 8(1.25 \mathrm{~g}, 4.0 \mathrm{mmol}), \mathrm{Pd}\left(\mathrm{PPh}_{3}\right)_{4}(70 \mathrm{mg}, 0.06$ mmol) and $\mathrm{Na}_{2} \mathrm{CO}_{3}(636 \mathrm{mg}, 6.0 \mathrm{mmol})$ were used. TPE-TPAPBI was obtained as yellowish green solid in $87 \%$ yield $(1.47 \mathrm{~g}) .{ }^{1} \mathrm{H} \mathrm{NMR}\left(600 \mathrm{MHz}, \mathrm{CD}_{2} \mathrm{Cl}_{2}\right), \delta(\mathrm{ppm}): 7.83-7.81\left(\mathrm{dd}, J_{1}=7.8 \mathrm{~Hz}, J_{2}=2.4 \mathrm{~Hz}\right.$, 1H), 7.61-7.59 (m, 2H), 7.54-7.48 (m, 5H), 7.45-7.43 (m, 2H), 7.40-7.30 (m, 7H), 7.28-7.24 (m, 6H), 7.16-7.05 (m, 20H), 7.04-7.01 (m, 2H). ${ }^{13} \mathrm{C}$ NMR (150 MHz, $\left.\mathrm{CD}_{2} \mathrm{Cl}_{2}\right), \delta$ (ppm): 153.3, 148.9, 148.5, $145.1,144.8,144.5,143.6,142.5,142.3,141.8,139.8,139.6,139.1,139.0,138.8,138.4,135.6,133.1$, 133.0, 132.6, 131.2, 131.1, 130.6, 130.2, 129.9, 129.1, 129.0, 128.8, 128.7, 127.9, 127.8, 127.7, 127.4, 127.3, 127.0, 126.8, 125.7, 125.1, 124.5, 124.3, 124.1, 120.9, 111.7. HRMS $\left(\mathrm{C}_{63} \mathrm{H}_{45} \mathrm{~N}_{3}\right): m / z 843.3633$ $\left[\mathrm{M}^{+}\right.$, calcd 843.3613].

1,2-Diphenyl-1,2-bis(4'-(1-phenyl-1H-benzo[d] imidazol-2-yl)-[1,1'-biphenyl]-4-yl)ethene $(T P E-$ DPBI). This compound was synthesized in a procedure similar to that for compound $\mathbf{9}$, in which 2 (980 mg, $2.0 \mathrm{mmol}), 8(1.88 \mathrm{~g}, 6.0 \mathrm{mmol}), \mathrm{Pd}\left(\mathrm{PPh}_{3}\right)_{4}(139 \mathrm{mg}, 0.12 \mathrm{mmol})$, and $\mathrm{Na}_{2} \mathrm{CO}_{3}(848 \mathrm{mg}, 8.0 \mathrm{mmol})$ were used. TPE-DPBI was obtained as yellowish green solid in $80 \%$ yield (1.39 g). ${ }^{1} \mathrm{H} \mathrm{NMR} \mathrm{(600} \mathrm{MHz,}$ $\left.\mathrm{CD}_{2} \mathrm{Cl}_{2}\right), \delta(\mathrm{ppm}): 7.82-7.81(\mathrm{~m}, 2 \mathrm{H}), 7.61-7.58(\mathrm{~m}, 4 \mathrm{H}), 7.54-7.47(\mathrm{~m}, 10 \mathrm{H}), 7.40-7.36(\mathrm{~m}, 4 \mathrm{H}), 7.35-$ $7.30(\mathrm{~m}, 6 \mathrm{H}), 7.27-7.22(\mathrm{~m}, 4 \mathrm{H}), 7.16-7.09(\mathrm{~m}, 12 \mathrm{H}), 7.07-7.06(\mathrm{~m}, 2 \mathrm{H}) .{ }^{13} \mathrm{C} \mathrm{NMR}\left(150 \mathrm{MHz}, \mathrm{CD}_{2} \mathrm{Cl}_{2}\right)$, $\delta$ (ppm): 153.3, 145.0, 144.9, 144.7, 144.6, 144.5, 142.5, 142.1, 139.2, 139.1, 138.8, 138.4, 133.1, 132.6, 
$131.2,131.1,130.3,129.9,129.1,129.0,128.8,127.9,127.8,127.7,127.5,127.3,124.5,124.1,120.9$,

111.7. HRMS $\left(\mathrm{C}_{64} \mathrm{H}_{44} \mathrm{~N}_{4}\right): m / z$ 868.3617 [M+, calcd 868.3566].

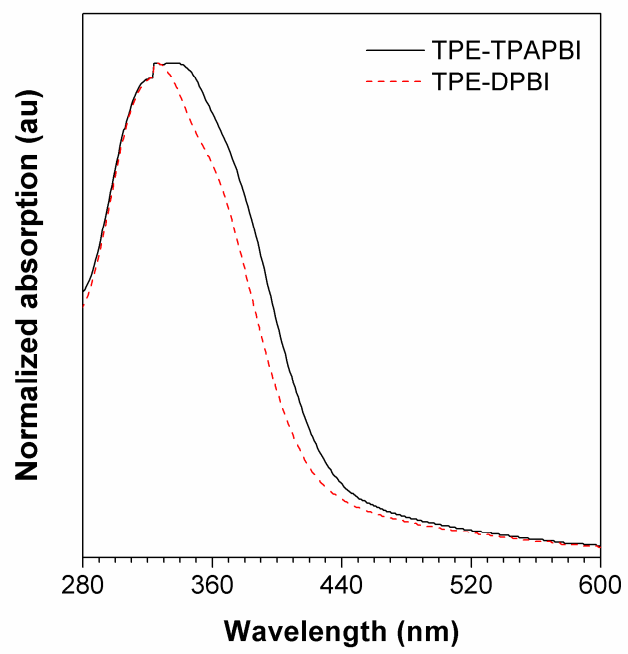

Figure S1. Absorption spectra of TPE-TPAPBI and TPE-DPBI in vacuum deposited films.

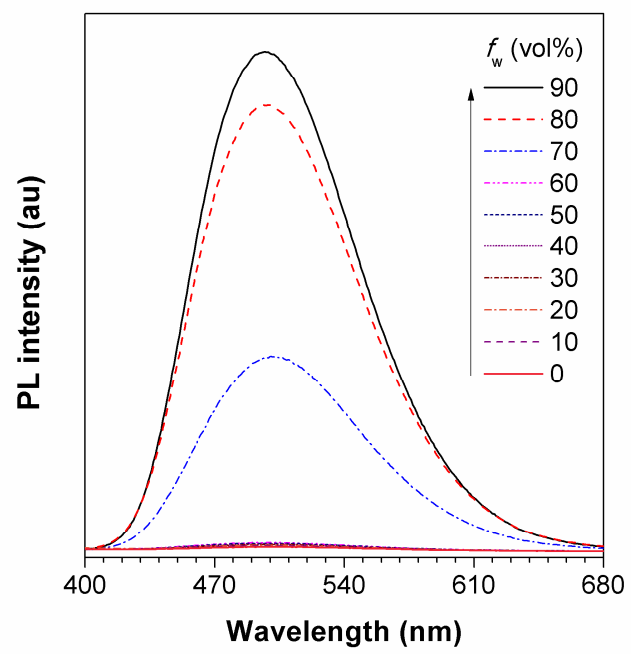

Figure S2. PL spectra of TPE-DPBI in THF/water mixtures with different water fractions $\left(f_{\mathrm{w}}\right)$. 

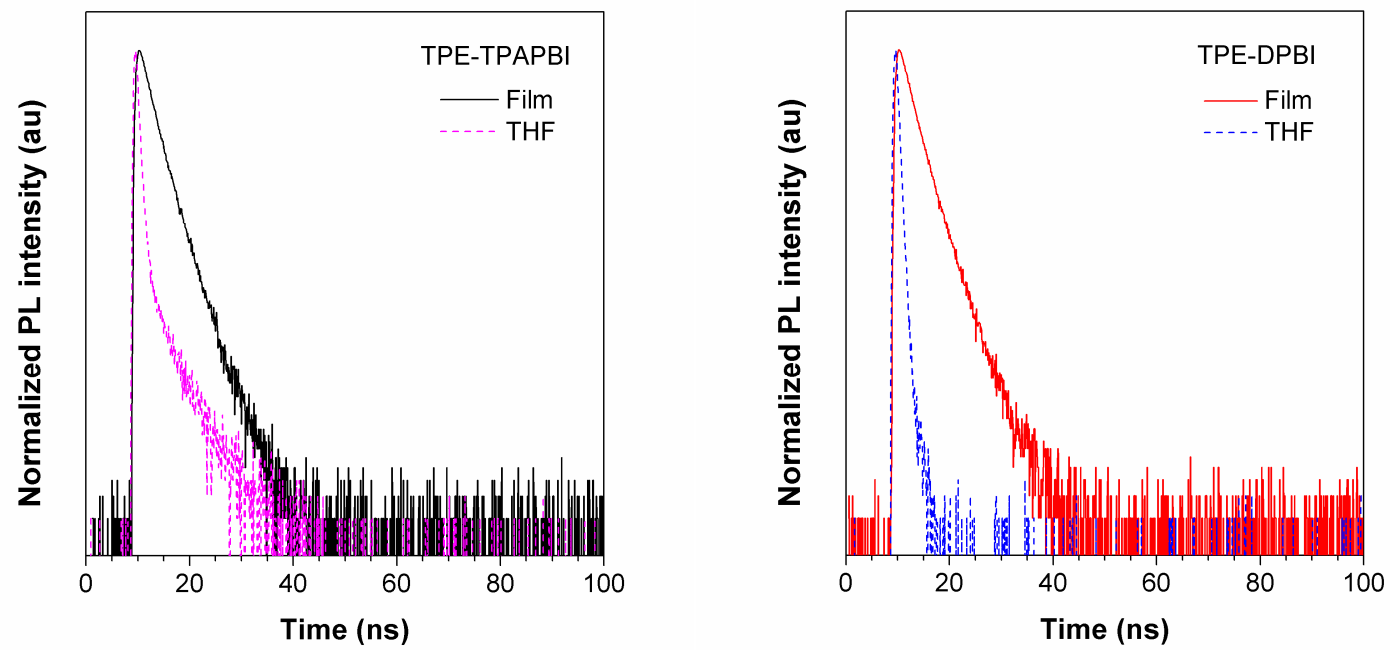

Figure S3. Fluorescence decay profiles of TPE-TPAPBI and TPE-DPBI in vacuum deposited films and THF.

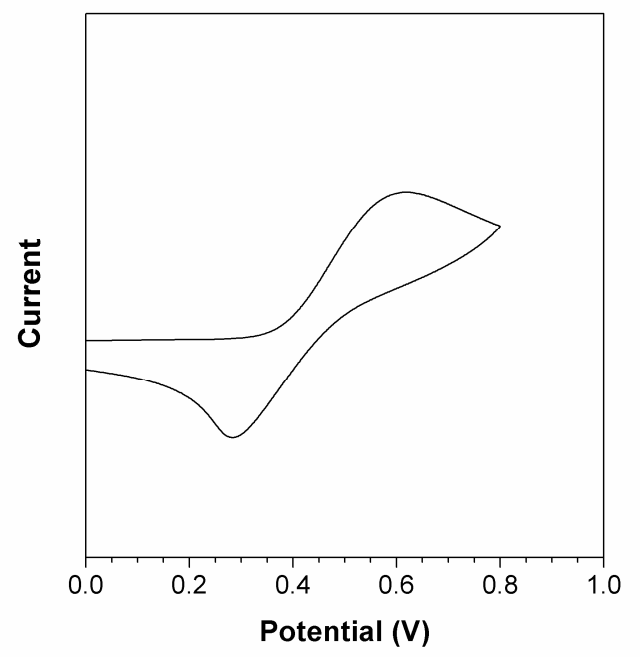

Figure S4. Cyclic voltammogram of ferrocene, measured in dichloromethane containing $0.1 \mathrm{M}$ tetra$n$-butylammonium hexafluorophosphate. Scan rate: $50 \mathrm{mV} \mathrm{s}^{-1}$. 


\section{References}

(1) Hu, R.; Maldonado, J. L.; Rodriguez, M.; Deng, C.; Jim, C. K. W.; Lam, J. W. Y.; Yuen, M. M. F.; Ramos-Ortiz, G.; Tang, B. Z. Luminogenic Materials Constructed from Tetraphenylethene Building Blocks: Synthesis, Aggregation-Induced Emission, Two-Photon Absorption, Light Refraction, and Explosive Detection. J. Mater. Chem. 2012, 22, 232-240.

(2) Wang, L.; Yang, X.; Wang, X.; Sun, L. Novel Organic Dyes with Anchoring Group of Quinoxaline-2,3-diol and the Application in Dye-Sensitized Solar Cells. Dyes Pigm. 2015, 113, 581-587.

(3) Ge, Z.; Hayakawa, T.; Ando, S.; Ueda, M.; Akiike, T.; Miyamoto, H.; Kajita, T.; Kakimoto, M.-A. Spin-Coated Highly Efficient Phosphorescent Organic Light-Emitting Diodes Based on Bipolar Triphenylamine-Benzimidazole Derivatives. Adv. Funct. Mater. 2008, 18, 584-590. 